\title{
About the Topographic Contrast in LVSEM
}

\section{J. Cazaux}

DTI, Faculty of Sciences, BP 1039, 51687, Reims Cedex 2, France

In $\mathrm{SEM}$, at primary beam energies $\mathrm{E}_{0}$ above $5 \mathrm{keV}$, the observed topographic contrast may easily be explained from the change of the SEE yield, $\delta$, with the angle of incidence, $i$, when standard Everhart-Thornley (E-T) detectors are used [1,2]. As shown in fig 1, a\&b (diamond sample) more complicated is to establish the same correlation in LVSEM $\left(\mathrm{E}_{0}<1 \mathrm{keV}\right)$ with an in-lens detector because the difficulty lies also in the architecture of the detection system.

The constant loss theory accounts fairly well for the yield from materials[1-3]. By a simple change of the range of incident electrons $\mathrm{R}$ into $\mathrm{R} / \cos \mathrm{i}$; it expresses the evolution of $\delta$ with i (fig $2 \mathrm{a} \&[4]$ ): $\delta\left(\mathrm{E}_{0}\right)=\mathrm{B}\left[\mathrm{E}_{0} / \mathrm{E}(\mathrm{e} . \mathrm{h})\right]$ [s/R cos i] [1-exp.-(Rcos i/s)] (1), [B: the escape probability; s: empirical attenuation length of the secondary electrons; E(e.h): energy required for generating an electronhole pair]. For Rcos $i>>s$ (or $E_{0}>5 \mathrm{keV}$ ), eq. 1 may be written in the form $\delta(i)=\delta(0) / \cos i(2)$ which accounts for the topographic contrast obtained with a E-T detector. When $\mathrm{E}_{0}$ is decreased down to $\mathrm{R}<<\mathrm{s}, \delta(\mathrm{i})$ takes the linear form, independent from $\mathrm{i},: \delta(\mathrm{i})=\mathrm{B}\left[\mathrm{E}_{0} / \mathrm{E}(\mathrm{e} . \mathrm{h})\right]$ which may explain the decrease in contrast previously observed with this type of detector [5]. In addition, the role of the emission angle $\alpha$ has to be taken into account when in-lens detectors are used (fig. $2 b$ ). This role may explains the main contrast obtained at 0.2 and at $0.5 \mathrm{keV}$ but the remaining difference between the two images (indicated by arrows in fig $1 ; 0.2 \mathrm{keV}$ in) require a deeper analysis.

To go deeper into the subject, various instrumental and theoretical causes may be considered. In modern instruments (ex.: fig 3a), an efficient in-lens detection is associated to a cross-over position being a function of the primary beam energy and of the working distance[6]. A non-Lambert-type emission of the SE's (fig 2c) may be due to a residual electric field on the specimen surface, to charging effects (insert fig 3a) but also to the physics of the SE emission induced by sub-KeV beams. In particular, the transport of the SEs in the specimen is less simple than suggested by eq.1; it involves isotropic random walks that may lead to escape probabilities, B, larger than 50\% (fig.3b $\&$ [7]). Sophisticated electron optical calculations for the SE trajectories in and out of the specimen are always possible but the first step is to control the stability of the microscope. For this goal, the use of the scatter diagram technique seems very promising (fig 1d \& [4]) and it may also be applied to two images acquired after an azimuth rotation (to control the axis-symmetry of the equipment).

\section{References}

[1]. D.C. Joy, J. Microscopy, 147 (1987) 51

[2] H. Seiler, .J. Appl. Phys. 53 (1983) R1

[3] J. Cazaux, J. Appl. Phys. 89(2001) 8265

[4] J.M. Patat et al, Scanning, in press.

[5] D.C Joy \& C.S Joy, Micron. 27(1996)247

[6] E. Plies. in Proc. $12^{\text {th }}$ EUREM, Brno, Czech Rep., P. Tomanek \& V. Kolarik ed. vol I,(2000)423

[7] J. Cazaux, Nucl. Instr. \& Meth in Phys. B ,in press. 


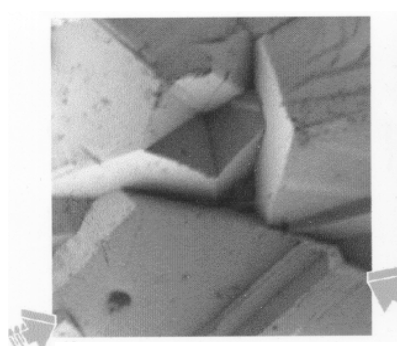

$\mathrm{a}: \mathrm{E}_{0}=0.2 \mathrm{keV}$ (in.)

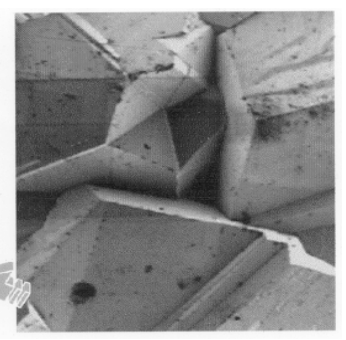

b: $\mathbf{E}_{0}=0.5 \mathrm{keV}$

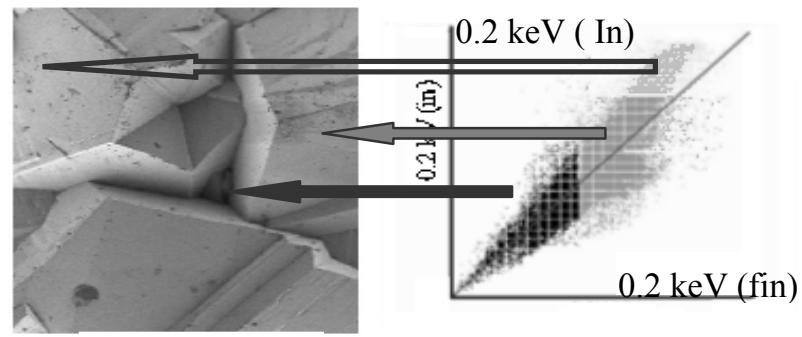

d: axb scat. diag

FIG 1a\&b: Obtained at $\mathrm{E}_{0}=0.2$ and $0.5 \mathrm{keV}$ (left) with a SEM (LEO, Gemini DSM 982:in-lens detection; magnification x1000; w.d.: $2.5 \mathrm{~mm}$ ), images of a micro-structured diamond specimen. After a series up to $E_{0}=15 \mathrm{keV}$, a final image, $c$, has also been taken at $0.2 \mathrm{kV}$ (fin). $\mathrm{d}$ : use of the scatter diagram technique for correlating the initial and the final images acquired at $0.2 \mathrm{kV}$ [4].

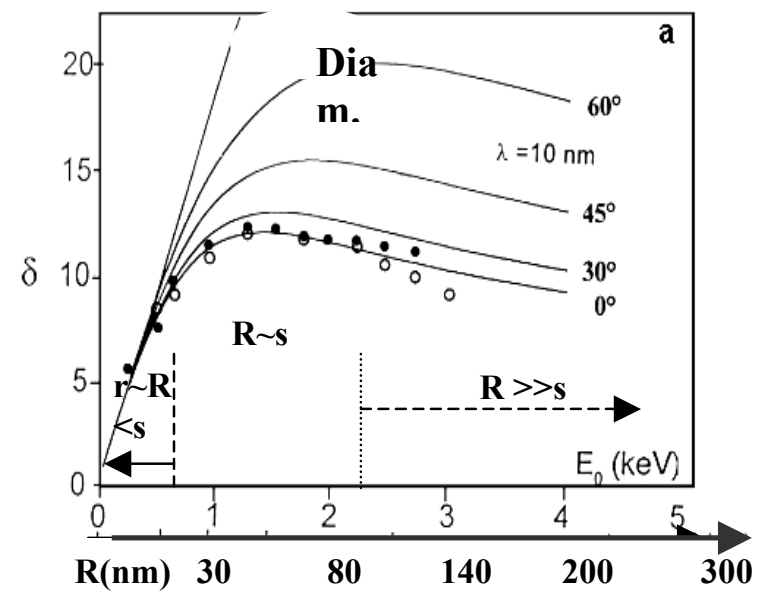

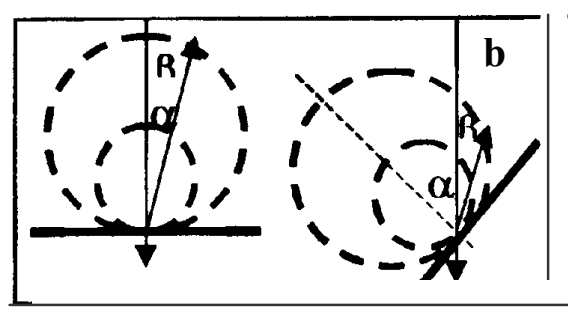

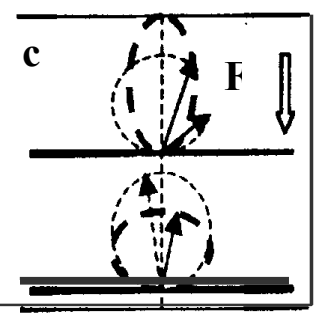

FIG 2. a(left):From eq. (1) with B 0.375; E(e.h) $=17$ $\mathrm{eV} ; \mathrm{s} \approx 22 \mathrm{~nm}$, calculated yield(curves), $\delta\left(\mathrm{E}_{0}\right)$ of a diamond specimen at various incidences compared to experiments (symbols)[3,4].

FIG $2 b$ (medium): Influence of the detection angles $\alpha \& \beta$ in the case of a Lambert emission. $\mathrm{c}$ (right) :possible causes of deviation of a $\cos \alpha$ emission.
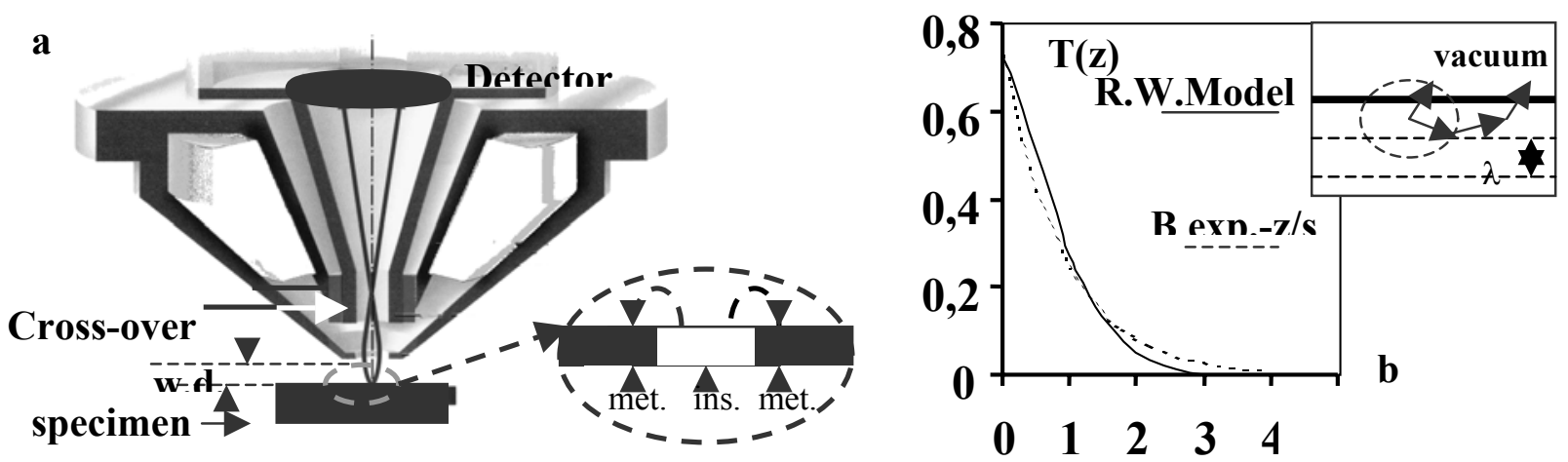

FIG. 3.a(left):Influence of the architecture of the instrument on the SE detection efficiency; insert: local field effect in differential charging. b(right): Comparison between SE transmission functions, $\mathrm{T}(\mathrm{z})$, based on an exponential decay (dashed line) and on a random walk model, R.W.M, involving a transport mean free path $\lambda$. Note that $\mathrm{T}(0)$ (or B) is $>50 \%[7]$. 